\title{
Assistência pré-natal em serviços públicos de saúde do Estado do Paraná
}

\author{
Prenatal care in public health services in the State of Paraná \\ Atención prenatal en los servicios de salud pública del Estado de Paraná
}

Lailla Ketly Ferreira Tiradentes Ruiz

ORCID: https://orcid.org/0000-0003-2650-2255

Universidade Estadual de Londrina, Brasil E-mail: laillaftruiz@gmail.com

Juliana Vicente de Oliveira Franchi

ORCID: https://orcid.org/0000-0001-9441-7736

Universidade Estadual de Londrina, Brasil E-mail: jolliveira@yahoo.com.br

Natália Carolina Rodrigues Colombo

ORCID: https://orcid.org/0000-0003-3770-8558

Universidade Estadual de Londrina, Brasil

E-mail: colombo_natalia@outlook.com

Fabiana Fontana Medeiros

ORCID: https://orcid.org/0000-0002-7876-572X

Universidade Estadual de Londrina, Brasil

E-mail: fontana.fabi@hotmail.com

Rosângela Aparecida Pimenta Ferrari

ORCID: https://orcid.org/0000-0003-0157-7461

Universidade Estadual de Londrina, Brasil

E-mail: ropimentaferrari@uel.br

Sandra Marisa Pelloso

ORCID: https://orcid.org/0000-0001-8455-6839

Universidade Estadual de Maringá, Brasil

E-mail: smpelloso@uem.br

Alexandrina Aparecida Maciel Cardelli

ORCID: http://orcid.org/0000-0002-0222-8821

Universidade estadual de Londrina, Brasil

E-mail: macielalexandrina@ hotmail.com

\begin{abstract}
Resumo
Objetivo: analisar a assistência pré-natal em serviços públicos de três Regionais de Saúde do Paraná. Metodologia: transversal, descritivo, quantitativo, recorte de uma coorte prospectiva, realizado em serviços de saúde da $9^{\mathrm{a}}$, da $10^{\mathrm{a}}$ e da $17^{\text {a. }}$ regionais, de julho de 2017 a março de 2018, com 1.211 mulheres. Utilizado prontuário materno, cartão de gestante e instrumento com informações socioeconômicas, demográficas e variáveis do pré-natal, agrupados e analisados estatisticamente no programa SPSS v20.0. Resultados: predominância de mulheres entre 19 e 34 anos ( $78,1 \%$ na $9^{\mathrm{a}}, 80,5 \%$ na $10^{\mathrm{a}}$ e $77,6 \%$ na $17^{\mathrm{a}}$ ); renda familiar de 1 a 3 salários mínimos $\left(67,8 \%\right.$ na $9^{\mathrm{a}}$., $62,9 \%$ na $10^{\mathrm{a}}$. e $63 \%$ na $\left.17^{\mathrm{a}}\right)$; $99 \%$ aderiram ao pré-natal nas 3 Regionais, com início até 14 semanas $\left(81,6 \%\right.$ na $9^{\mathrm{a}}$, $69.6 \%$ na $10^{\mathrm{a}}$. e $79,0 \%$ na $17^{\mathrm{a}}$ ); $80 \%$ das gestantes realizaram 6 ou mais consultas, com classificação de risco habitual $\left(65,5 \%\right.$ na $9^{\mathrm{a}}$, $62,8 \%$ na $10^{\mathrm{a}}$ e $53,1 \%$ na $17^{\mathrm{a}}$ ); baixas taxas de encaminhamentos a serviço especializado $\left(14,9 \%\right.$ na $9^{\mathrm{a}}$, $27,5 \%$ na $10^{\mathrm{a}}$. e $28,7 \%$ na $17^{\mathrm{a}}$ ) com tempo de espera maior $\left(47,2 \%\right.$ ) de 29 dias na $17^{\mathrm{a}}$; na $17^{\mathrm{a}}$ destaca-se baixa realização de citologia oncótica e consulta odontológica em apenas $59,5 \%$ e $63,2 \%$, respectivamente. Conclusão: a assistência pré-natal nos serviços públicos de saúde, de modo geral, atende às determinações do Ministério da Saúde, porém, faz-se necessário que os gestores proporcionem capacitações assertivas aos profissionais envolvidos, para implementação adequada dos protocolos nacionais visando desfechos positivos do período gravídico-puerperal.
\end{abstract}

Palavras-chave: Saúde materna; Cuidado pré-natal; Qualidade da assistência à saúde.

\begin{abstract}
Objective: to analyze prenatal care in public services of three Health Regional in Paraná. Methodology: crosssectional, descriptive, quantitative, cut from a prospective cohort, performed in health services of the 9th, 10th. and the $17^{\text {th }}$ Regional from July 2017 to March 2018, with 1,211 women. Maternal medical records, pregnant women card and instrument with socioeconomic and demographic data, and prenatal variables were used, grouped, and analyzed statistically in the SPSS v20.0 program. Results: predominance of women between 19 and 34 years old $(78.1 \%$ in the $9^{\text {th }}, 80.5 \%$ in the $10^{\text {th }}$ and $77.6 \%$ in the $\left.17^{\text {th }}\right)$; family income of 1 to 3 minimum wages $\left(67.8 \%\right.$ on the $9^{\text {th }}, 62.9 \%$ on the $10^{\text {th }}$ and $63 \%$ on the $\left.17^{\text {th }}\right) ; 99 \%$ adhered to prenatal care in the 3 Regional, beginning within 14 weeks $(81.6 \%$ on the
\end{abstract}


$9^{\text {th }}, 69.6 \%$ on the $10^{\text {th }}$ and $79.0 \%$ on the $\left.17^{\text {th }}\right) ; 80 \%$ of pregnant women had 6 or more consultations, with the usual risk classification $\left(65.5 \%\right.$ on the $9^{\text {th }}, 62.8 \%$ on the $10^{\text {th }}$ and $53.1 \%$ on the $\left.17^{\text {th }}\right)$; low rates of referrals to specialized services $\left(14.9 \%\right.$ on the $9^{\text {th }}, 27.5 \%$ on the $10^{\text {th }}$ and $28.7 \%$ on the $\left.17^{\text {th }}\right)$, with a waiting time longer than 29 days on the $17^{\text {th }}(47.2 \%)$; in the 17 th, low performance of oncotic cytology and dental consultation stands out, with only $59.5 \%$ and $63.2 \%$, respectively. Conclusion: prenatal care in public health services, in general, meets the requirements of the Ministry of Health, however, it is necessary that managers provide assertive training to the professionals involved, for the proper implementation of national protocols aiming at positive outcomes of the pregnancy-puerperal period.

Keywords: Maternal health; Prenatal care; Quality of health care.

\section{Resumen}

Objetivo: analizar la atención prenatal en los servicios públicos de tres Regionales de Salud del Paraná. Metodología: descriptivo transversal, cuantitativo, recorte de una cohorte prospectiva, realizado en servicios de salud de la $9^{\mathrm{a}}, 10^{\mathrm{a}} \mathrm{y}$ $17^{\mathrm{a}}$ Regional de Salud de julio de 2017 a marzo de 2018, con 1.211 mujeres. Se utilizaron registros médicos maternos, tarjeta de gestantes e instrumento con informaciones socioeconómicas y demográficas y variables prenatales, agrupados y analizados estadísticamente en el programa SPSS v20.0. Resultados: predominio de mujeres de 19 a 34 años (78,1\% en la $9^{a}$ Regional, $80,5 \%$ en la $10^{a}$ Regional y 77,6\% en la $17^{\text {a }}$ Regional), renta familiar de 1 a 3 salarios (67,8\% en la $9^{\mathrm{a}}$ Regional, $62,9 \%$ en la $10^{\mathrm{a}}$ Regional y $63 \%$ en la $17^{\mathrm{a}}$ Regional); $99 \%$ se adhirió a la atención prenatal en las 3 Regionales, a partir de las 14 semanas (81,6\% en la $9^{\mathrm{a}}$ Regional, $69,6 \%$ en la $10^{\mathrm{a}}$ Regional y $79,0 \%$ en la $17^{\mathrm{a}}$ Regional); $80 \%$ de las embarazadas realizaron 6 o más consultas, con la clasificación de riesgo habitual (65,5\% en la $9^{\mathrm{a}}$ Regional, $62,8 \%$ en la $10^{\mathrm{a}}$ Regional y $53,1 \%$ en la $17^{\mathrm{a}}$ Regional); bajas tasas de orientación a servicio especializado ( $14,9 \%$ en la $9^{\mathrm{a}}$ Regional, $27,5 \%$ en la $10^{\mathrm{a}}$ Regional y $28,7 \%$ en la $17^{\mathrm{a}}$ Regional), con tiempo de espera superior a 29 días en la $17^{\mathrm{a}}$ Regional (47,2\%); en la $17^{\mathrm{a}}$ Regional, se destaca la baja realización de citología oncótica y de consulta odontológica, con solamente 59,5\% y $63,2 \%$, respectivamente. Conclusión: la atención prenatal en los servicios de salud pública, en general, acata los requisitos del Ministerio de Salud, sin embargo, se hace necesario que los gestores ofrezcan capacitaciones asertivas a los profesionales involucrados, para la implementación adecuada de los protocolos nacionales, aspirando a la conclusión positiva del período embarazo-puerperal.

Palabras clave: Salud materna; Atención prenatal; Cualidad de la atención de salud.

\section{Introdução}

O pré-natal é a assistência voltada ao momento gravídico-puerperal que, por meio de ações planejadas, visa promover o cuidado integral e identificar possíveis mudanças fisiológicas e psicológicas. Dessa forma, possibilita a minimização dos riscos que possam vir a se transformar em algo negativo à mulher e a seu filho, proporcionando, ao momento vivido, algo natural, prazeroso e único (Cardoso, Júnior, Santos, Santos \& Cariri, 2017).

A cada ano, aumenta a preocupação dos profissionais da área em relação à adequação do pré-natal, uma vez que os agravos, durante a gestação, têm contribuído para desfechos desfavoráveis. As principais intercorrências encontradas são infecção do trato urinário (ITU), doença hipertensiva exclusiva da gestação (DHEG), que favorecem, muitas vezes, ocorrências de partos prematuros e até mesmo eclampsias. Alguns fatores já foram comprovados, como agravantes dessas intercorrências, como baixo nível socioeconômico, faixa etária menor de 15 e maior de 35 anos e presença de comorbidades (Varela, Oliveira, Melo \& Mathias, 2017).

O pré-natal de qualidade deve acolher a mulher e sua família, promover ações resolutivas e contínuas, por meio da execução, acompanhamento e direcionamento ao atendimento especializado, quando necessário, contribuindo para a redução da mortalidade materno-infantil, que é grave problema de saúde pública mundial (Guimarães et al., 2018). Esse cuidado abrange muito além de executar consultas para verificação de peso e demais aferições obstétricas.

O Ministério da Saúde (MS), em 2010, voltado a proporcionar melhorias no atendimento gravídico puerperal, elaborou protocolos de atendimentos que padronizam e direcionam o atendimento dos profissionais envolvidos, conhecido como Programa de Humanização de Pré-Natal e Nascimento (PHPN), que, alguns anos após, em 2011, recebeu o nome de Rede Cegonha (Portaria n $\left.{ }^{\circ} 1.459,2011\right)$.

A Rede Cegonha $(\mathrm{RC})$ objetivou organizar a Rede de Atenção à Saúde Materna e Infantil, com ações voltadas à facilidade de acesso, acolhimento e resolutividade, além de redução da mortalidade materna e infantil (Portaria $\left.\mathrm{n}^{\circ} 1.459,2011\right)$. 
Quando algum determinante interfere no processo natural da gestação e favorece complicações para o binômio mãe/filho, considera-se esta gravidez de risco. Uma maneira de identificar agravos e agir em tempo oportuno é o início precoce do seguimento pré-natal, momento em que todo profissional envolvido nesse cuidado necessita ser capacitado e proativo a fim de identificar elementos sinalizadores de complicações (Santos \& Soares, 2018). Os agravos na gestação podem requerer internações e, nesse período, é certamente sinal alarmante pois a mulher encontra-se num momento mais vulnerável, suscetível a desequilíbrios emocionais e físicos, predispondo-se a maiores complicações (Falavina, Oliveir, Melo, Varela \& Mathias, 2017).

Mediante o exposto, tomaram-se como base as determinações do MS (Portaria $\mathrm{n}^{\circ} 1.459,2011$ ) sobre a realização adequada do atendimento pré-natal, para atender ao objetivo deste estudo, que foi analisar esse cuidado em serviços públicos de saúde do Estado do Paraná.

\section{Metodologia}

Estudo quantitativo, transversal, descritivo, recorte de uma coorte prospectiva de um projeto multicêntrico intitulado: "Rede Mãe Paranaense, na perspectiva da usuária: o cuidado da mulher no pré-natal, no parto, no puerpério e da criança", financiado pelo Ministério da Ciência, Tecnologia e Inovação (MCTI) e Conselho Nacional de Desenvolvimento Científico e Tecnológico (CNPq) - chamada universal MCTI/CNPq 01/2016.

A população do estudo foi composta por mulheres atendidas nas maternidades de referências para o parto na Rede Mãe Paranaense (RMP) de três Regionais de Saúde (RS) sendo a $9^{\mathrm{a}}, 10^{\mathrm{a}}$ e $17^{\mathrm{a}}$, que atendem gestação de risco habitual, intermediário e alto risco. Com base no número de partos que ocorreram em 2016, a amostra resultou em 1211 mulheres (397 pertencentes à $9^{\mathrm{a}}$. RS, 385 à $10^{\mathrm{a}}$. RS e 429 à $17^{\mathrm{a}}$. RS), já com acréscimo de $20 \%$ como margem de segurança para possíveis perdas e nível de significância de 5\%.

Os critérios de inclusão foram mulheres que tiveram seus partos nos hospitais envolvidos, residentes da área urbana e com idade gestacional maior ou igual a 32 semanas de gestação. A coleta de dados ocorreu no período de julho de 2017 a março de 2018 em hospitais referência para risco habitual, intermediário e alto risco da $9^{\text {a. }}$ RS da região de Foz do Iguaçu (Hospital A), 10. RS da região de Cascavel (Hospital B) e 17. RS de Londrina (Hospital C, D, E, F e G), totalizando 55 municípios envolvidos na pesquisa.

Foram utilizadas informações do prontuário materno, carteira de saúde das gestantes e entrevista com as puérperas ainda na maternidade, com pelo menos 24 horas após o parto.

Para a entrevista, foi aplicado um instrumento com questões que englobavam informações socioeconômicas e demográficas das mulheres bem como variáveis relacionadas ao pré-natal atual, adesão ao pré-natal e local de realização; cadastramento no Sistema de Informatização em Saúde pré-natal (SIS pré-natal); número total de consultas; consultas odontológicas; citologia oncótica (exame para detecção de câncer de colo de útero); cobertura vacinal; acolhimento e encaminhamentos às intercorrências gestacionais; classificação de risco (habitual, intermediário e alto risco); participação em grupos de gestantes; intervalo entre a última consulta pré-natal e o parto.

Utilizaram-se os programas Microsoft Excel ${ }^{\circledR}$ e Statistical Package for Social Sciences (SPSS), versão 20.0 para análise descritiva dos dados. Este tipo de análise oferece um retrato específico em tempo e espaço sobre o objeto de estudo, bem como a possibilidade de comparações com realidades afins (Pereira, Shitsuka, Parreira \& Shitsuka, 2018).

Esta pesquisa foi submetida e aprovada pelo Comitê de Ética da Universidade Estadual de Londrina (CEP/UEL) com número 2.053.304 CAAE: 67574517.1.1001.5231, em 9 de maio de 2017. 


\section{Resultados}

A caracterização sociodemográfica das gestantes nas três RS demonstrou predominância de mulheres adultas jovens entre 19 e 34 anos $\left(78,1 \%\right.$ na $9^{\text {a. }}, 80,5 \%$ na $10^{\text {a. }}$ e $77,6 \%$ na $17^{\mathrm{a}}$.), branca $\left(53,4 \%\right.$ na $9^{\mathrm{a}}, 63,6 \%$ na $10^{\mathrm{a}}$ e $60 \%$ na $17^{\mathrm{a}}$. $)$ e com companheiro $\left(90,2 \%\right.$ na $9^{\mathrm{a}}$, $90,9 \%$ na $10^{\mathrm{a} .}$ e $85,8 \%$ na $17^{\mathrm{a}}$ ), grau de escolaridade nível 3 (abrange nível médio completo ou mais) em menos da metade das mulheres nas três RSs (Tabela 1).

Em relação a situação econômica, em torno de $60 \%$ das mulheres nas 3 RSs apresentavam renda familiar de 1 a 3 salários mínimos e não eram beneficiárias de programas do governo em $80 \%$ da amostra em cada RS (Tabela 1).

Tabela 1. Perfil socioeconômico e demográfico das gestantes atendidas nos serviços públicos de Saúde do Estado do Paraná, PR, 2020.

\begin{tabular}{|c|c|c|c|c|c|c|}
\hline \multirow{3}{*}{ Variáveis } & \multicolumn{6}{|c|}{ Regionais de Saúde } \\
\hline & \multicolumn{2}{|c|}{$9^{\text {a. }}$} & \multicolumn{2}{|c|}{$10^{\mathrm{a} .}$} & \multicolumn{2}{|c|}{$17^{\mathrm{a}}$} \\
\hline & $\mathbf{n}$ & $\%$ & $\mathbf{n}$ & $\%$ & $\mathbf{n}$ & $\%$ \\
\hline \multicolumn{7}{|l|}{ Idade (em anos) } \\
\hline Até 18 & 42 & 10,6 & 23 & 6,0 & 55 & 12,8 \\
\hline 19 a 34 & 310 & 78,1 & 310 & 80,5 & 333 & 77,6 \\
\hline 35 e mais & 45 & 11,3 & 52 & 13,5 & 41 & 9,6 \\
\hline \multicolumn{7}{|l|}{ Raça/cor } \\
\hline Branca & 212 & 53,4 & 245 & 63,6 & 257 & 60,0 \\
\hline Negra & 32 & 8,1 & 19 & 5,0 & 39 & 9,0 \\
\hline Outro (pardo, índio, amarelo) & 153 & 38,5 & 121 & 31,4 & 133 & 31,0 \\
\hline \multicolumn{7}{|l|}{ Situação Conjugal } \\
\hline Com companheiro & 358 & 90,2 & 350 & 90,9 & 368 & 85,8 \\
\hline Sem companheiro & 39 & 9,8 & 35 & 9,1 & 61 & 14,2 \\
\hline \multicolumn{7}{|l|}{ Escolaridade * } \\
\hline Nível 1 & 90 & 22,7 & 87 & 22,7 & 90 & 20,2 \\
\hline Nível 2 & 117 & 29,5 & 115 & 29,9 & 141 & 33,2 \\
\hline Nível 3 & 190 & 47,8 & 183 & 47,4 & 198 & 46,6 \\
\hline \multicolumn{7}{|l|}{ Condição de ocupação } \\
\hline Remunerada & 182 & 45,8 & 197 & 51,2 & 172 & 40,0 \\
\hline Não remunerada & 215 & 54,2 & 188 & 48,8 & 257 & 60,0 \\
\hline \multicolumn{7}{|c|}{ Renda Familiar (em salários mínimo)** } \\
\hline Menos 1 & 32 & 8,1 & 65 & 16,9 & 86 & 20,0 \\
\hline De 1 a 3 & 269 & 67,8 & 242 & 62,9 & 270 & 63,0 \\
\hline Mais que 3 & 96 & 24,1 & 78 & 20,2 & 73 & 17,0 \\
\hline \multicolumn{7}{|l|}{ Benefício governo } \\
\hline Sim & 60 & 15,1 & 44 & 11,0 & 90 & 21,0 \\
\hline Não & 337 & 84,9 & 341 & 89,0 & 339 & 79,0 \\
\hline Total & 397 & 100,0 & 385 & 100,0 & 429 & 100,0 \\
\hline
\end{tabular}

* Nível 1 - até Ensino Fundamental incompleto, Nível 2 - abrange Ensino Fundamental completo a Médio incompleto,

Nível 3 - abrange Ensino Médio completo a Superior incompleto ou mais

* Fonte: Ministério da Educação (2020)

** salário mínimo de 2017 - R\$937,00

Fonte: Dados da pesquisa. 
Quanto ao pré-natal atual, quase a totalidade teve adesão a esse cuidado nas regionais analisadas, sendo que a maior parte no sistema público (Unidade Básica de Saúde - UBS e/ou ambulatório) e classificadas como risco habitual (65,5\% na 9a., $62,8 \%$ na $10^{\mathrm{a}}$ e $53,1 \%$ na $17^{\mathrm{a}}$ ). A informação sobre risco foi fornecida a pouco mais da metade das mulheres nas três RSs, sendo pelo médico na $10^{\mathrm{a}}$. RS e na $17^{\mathrm{a}}$. RS (em torno de $70 \%$ ) e pelo enfermeiro e equipe de enfermagem na $9^{\mathrm{a}}$. RS $(61,6 \%$ ). Entre as mulheres classificadas como risco intermediário e alto risco, nas três RSs, houve continuidade de acompanhamento na UBS de origem com a maior parte das gestantes $\left(89,8 \%\right.$ na $9^{\mathrm{a}}$, $70,6 \%$ na $10^{\mathrm{a}}$ e $85,6 \%$ na $17^{\mathrm{a}}$ ). Vale ressaltar que muitas mulheres $\left(85,1 \%\right.$ na $9^{\mathrm{a}}$, $72,5 \%$ na $10^{\mathrm{a}}$. e $71,3 \%$ na $17^{\mathrm{a}}$ ) não tiveram encaminhamento a serviço especializado; já as que foram encaminhadas, mencionaram tempo de espera de até 7 dias na $9^{\text {a. }}$ RS e na $10^{\text {a. }}$ RS ( $61 \%$ e $58,4 \%$, respectivamente) ou de mais de 29 dias na $17^{\text {a. }}$ RS $(47,2 \%)$ e $80 \%$ das mulheres nas três RSs não participaram dos grupos para gestantes (Tabela 2).

Tabela 2. Caracterização da assistência pré-natal nos serviços públicos de Saúde do Estado do Paraná, PR, 2020.

\begin{tabular}{|c|c|c|c|c|c|c|}
\hline \multirow{3}{*}{ Variáveis } & \multicolumn{6}{|c|}{ Regionais de Saúde } \\
\hline & \multicolumn{2}{|c|}{$9^{\mathbf{a}}$} & \multicolumn{2}{|c|}{$10^{\mathrm{a}}$} & \multicolumn{2}{|c|}{$17^{\mathrm{a}}$} \\
\hline & $\mathbf{n}$ & $\%$ & $\mathbf{n}$ & $\%$ & $\mathbf{n}$ & $\%$ \\
\hline \multicolumn{7}{|l|}{ Realização Pré-Natal } \\
\hline Sim & 394 & 99,2 & 381 & 99,0 & 427 & 99,5 \\
\hline Não & 3 & 0,8 & 4 & 1,0 & 2 & 0,5 \\
\hline \multicolumn{7}{|l|}{ Local Pré-Natal } \\
\hline Público & 365 & 92,6 & 299 & 77,7 & 334 & 78,6 \\
\hline Público com particular ou convênio & 32 & 7,4 & 86 & 22,3 & 95 & 21,4 \\
\hline \multicolumn{7}{|l|}{ Classificação Risco } \\
\hline Risco Habitual & 260 & 65,5 & 242 & 62,8 & 228 & 53,1 \\
\hline Risco Intermediário & 55 & 13,9 & 20 & 5,3 & 105 & 24,1 \\
\hline Alto Risco & 82 & 20,6 & 123 & 31,9 & 96 & 22,8 \\
\hline \multicolumn{7}{|l|}{ Informação sobre risco } \\
\hline Sim & 224 & 56,6 & 225 & 58,6 & 259 & 60,8 \\
\hline Não & 173 & 43,4 & 160 & 41,4 & 170 & 39,2 \\
\hline \multicolumn{7}{|l|}{ Profissional que informou risco } \\
\hline Enfermeiro / Equipe Enfermagem & 138 & 61,6 & 50 & 22,2 & 58 & 22,4 \\
\hline Médico & 86 & 38,4 & 175 & 77,8 & 201 & 77,6 \\
\hline \multicolumn{7}{|l|}{ Continuidade da assistência na UBS } \\
\hline Sim & 123 & 89,8 & 101 & 70,6 & 172 & 85,6 \\
\hline Não & 14 & 10,2 & 42 & 29,4 & 29 & 14,4 \\
\hline \multicolumn{7}{|l|}{$\begin{array}{l}\text { Encaminhamento a serviço } \\
\text { especializado }\end{array}$} \\
\hline Sim & 59 & 14,9 & 106 & 27,5 & 123 & 28,7 \\
\hline Não & 338 & 85,1 & 279 & 72,5 & 306 & 71,3 \\
\hline \multicolumn{7}{|l|}{$\begin{array}{l}\text { Tempo espera serviço especializado } \\
\text { (em dias) }\end{array}$} \\
\hline Rápido/Imediato & 20 & 33,9 & 24 & 22,6 & 10 & 8,1 \\
\hline 2 a 7 & 16 & 27,1 & 38 & 35,8 & 24 & 19,5 \\
\hline 8 a 28 & 18 & 30,5 & 22 & 20,8 & 31 & 25,2 \\
\hline acima de 29 & 5 & 8,5 & 22 & 20,8 & 58 & 47,2 \\
\hline & 5 & & & & & \\
\hline
\end{tabular}




\section{Participação grupo de gestantes}

\begin{tabular}{lrrrrrr} 
Sim & 67 & 16,9 & 72 & 18,7 & 79 & 18,4 \\
Não & 330 & 83,1 & 313 & 81,3 & 350 & 81,6 \\
\hline Total & 397 & 100,0 & 385 & 100,0 & 429 & 100,0 \\
\hline
\end{tabular}

Fonte: Dados da pesquisa.

A análise das etapas da assistência pré-natal demonstrou predominância de cadastro SIS pré-natal na $10^{\mathrm{a}}$. RS (93,4\%), início da assistência antes de 14 semanas de gestação, totalizando em 6 ou mais consultas em mais de $80 \%$ da amostra nas três RSs. O profissional enfermeiro foi responsável pela maioria delas $\left(85,6 \%\right.$ na $9^{\mathrm{a}}$, $81,5 \%$ na $10^{\mathrm{a}}$ e $74,6 \%$ na $17^{\mathrm{a}}$ ) das primeiras consultas (Tabela 3).

Ainda nas etapas do pré-natal, grande parte das mulheres cumpriu com a imunização de acordo com o calendário vacinal disponível a este grupo. Foi perceptível que na $9^{\mathrm{a}}$. RS $(60,2 \%)$ e na $10^{\mathrm{a}}$. RS $(66,8 \%)$, houve maior porcentagem de gestantes que não realizaram a citologia oncótica durante período gestacional ou no intervalo menor de 1 ano. Quanto à participação das gestantes em consultas odontológicas, houve destaque apenas na $17^{\mathrm{a}} \cdot \mathrm{RS}(63,2 \%)$, e o intervalo entre a última consulta PN e o parto, nas três RSs, foi inferior a 15 dias (72\% na 9 $9^{\mathrm{a}}, 86,0 \%$ na $10^{\mathrm{a}}$. e 83,7\% na $17^{\mathrm{a}}$ ) (Tabela 3$)$.

Tabela 3. Assistência pré-natal nos serviços púbicos de Saúde do Estado do Paraná, PR, 2020.

\begin{tabular}{|c|c|c|c|c|c|c|}
\hline \multirow{3}{*}{ Variáveis } & \multicolumn{6}{|c|}{ Regionais de Saúde } \\
\hline & \multicolumn{2}{|c|}{$9^{\mathrm{a}}$} & \multicolumn{2}{|c|}{$10^{\mathrm{a} .}$} & \multicolumn{2}{|c|}{$17^{\mathrm{a} .}$} \\
\hline & $\mathbf{n}$ & $\%$ & $\mathbf{n}$ & $\%$ & $\mathbf{n}$ & $\%$ \\
\hline \multicolumn{7}{|l|}{ Cadastro SIS Pré-Natal } \\
\hline Sim & 353 & 89,1 & 355 & 93,4 & 313 & 69,3 \\
\hline Não & 9 & 2,3 & 16 & 3,4 & 59 & 12,3 \\
\hline Não sabe informar & 35 & 8,6 & 14 & 3,2 & 57 & 13,0 \\
\hline \multicolumn{7}{|c|}{ Início Pré-Natal (em semanas) } \\
\hline antes $14^{\mathrm{a}}$ & 324 & 81,6 & 268 & 69,6 & 339 & 79,0 \\
\hline $14^{\mathrm{a}}$ a $27^{\mathrm{a}}$ & 62 & 15,6 & 95 & 24,7 & 83 & 19,4 \\
\hline mais $27^{\mathrm{a}}$ & 11 & 2,8 & 22 & 5,7 & 7 & 1,6 \\
\hline \multicolumn{7}{|l|}{$\mathbf{N}^{0}$ consultas Pré-Natal } \\
\hline 0 a 2 & 14 & 3,4 & 13 & 3,4 & 6 & 1,4 \\
\hline 3 a 5 & 58 & 14,4 & 31 & 8,0 & 56 & 13,1 \\
\hline 6 ou mais & 325 & 82,2 & 341 & 88,6 & 367 & 85,5 \\
\hline \multicolumn{7}{|l|}{$1^{a}$ Consulta Pré-Natal } \\
\hline Enfermeiro & 340 & 85,6 & 314 & 81,5 & 320 & 74,6 \\
\hline Médico & 48 & 12,1 & 56 & 14,6 & 76 & 17,7 \\
\hline Outros profissionais & 9 & 2,3 & 15 & 3,9 & 33 & 7,7 \\
\hline \multicolumn{7}{|l|}{ Vacina Antitetânica } \\
\hline Sim & 373 & 94,0 & 368 & 95,6 & 425 & 99,1 \\
\hline Não & 24 & 6,0 & 17 & 4,4 & 4 & 0,9 \\
\hline \multicolumn{7}{|l|}{ Vacina Anti-hepatite B } \\
\hline Sim & 375 & 94,5 & 339 & 88,1 & 417 & 97,2 \\
\hline Não & 22 & 5,5 & 46 & 11,9 & 12 & 2,8 \\
\hline
\end{tabular}




\section{Vacina Gripe}

\begin{tabular}{lrrrrrr} 
Sim & 372 & 93,7 & 352 & 91,4 & 402 & 93,7 \\
Não & 25 & 6,3 & 33 & 8,6 & 27 & 6,3 \\
Citologia Oncótica & & & & & & \\
Sim & 158 & 39,8 & 128 & 33,2 & 255 & 59,5 \\
Não & 239 & 60,2 & 257 & 66,8 & 174 & 40,5 \\
Consulta Odontológica & & & & & & \\
Sim & 142 & 35,8 & 162 & 42,2 & 271 & 63,2 \\
Não & 255 & 64,2 & 223 & 57,8 & 158 & 36,8 \\
Intervalo Pré-Natal e parto (em & & & & & & \\
dias) & & & & & & \\
$0-15$ & 285 & 72,0 & 331 & 86,0 & 359 & 83,7 \\
$16-45$ & 99 & 24,7 & 45 & 11,6 & 58 & 13,5 \\
$\geq 46$ & 13 & 3,3 & 9 & 2,4 & 12 & 2,8 \\
\hline Total & 397 & 100,0 & 385 & 100,0 & 429 & 100,0 \\
\hline
\end{tabular}

Fonte: Dados da pesquisa

Na Tabela 4, apresentam-se as intercorrências relacionadas à gestação, demonstrando que nas regionais analisadas, a maioria das gestantes $\left(56,7 \%\right.$ na $9^{\mathrm{a}}$, $65,5 \%$ na $10^{\mathrm{a}}$ e $68,3 \%$ na $17^{\mathrm{a}}$ ), não mencionaram intercorrências, entre as que apresentaram, as principais intercorrências foram sangramento $\left(9,8 \%\right.$ na $9^{\mathrm{a}}$, $4,9 \%$ na $10^{\mathrm{a} .}$ e $8,4 \%$ na $17^{\mathrm{a}}$ ), doença hipertensiva $\left(5,0 \%\right.$ na $9^{\mathrm{a}}, 7,0 \%$ na $10^{\mathrm{a}}$ e $8,2 \%$ na $17^{\mathrm{a}}$.) e doenças clínicas $\left(2,5 \%\right.$ na $9^{\mathrm{a}}, 3,1 \%$ na $10^{\mathrm{a}}$ e $2,1 \%$ na $\left.17^{\mathrm{a}}\right)$.

Dentre as que apresentaram intercorrências, buscaram atendimento principalmente em nível hospitalar (63,6\% na 9a., $74,6 \%$ na $10^{\text {a }}$ e $59,1 \%$ na $17^{\mathrm{a}}$ ), avaliando o atendimento como rápido e resolutivo $\left(92,4 \%\right.$ na $9^{\mathrm{a}}$, $89,8 \%$ na $10^{\mathrm{a}}$. e $64,2 \%$ na $17^{\mathrm{a}}$ ). Muitas das entrevistadas $\left(94,5 \%\right.$ na $9^{\mathrm{a}}$, $93,5 \%$ na $10^{\mathrm{a}}$ e $86,5 \%$ na $17^{\mathrm{a}}$ ), nas três RS, relataram terem sido orientadas quanto ao hospital que deveriam buscar no caso de intercorrências.

Em relação à opinião das gestantes quanto ao nível de satisfação com o atendimento, observou-se que grande parte demonstrou satisfação com a assistência pré-natal recebida (80,6\% na $9^{\mathrm{a}}$., $90,6 \%$ na $10^{\mathrm{a}}$. e 82,3\% na $17^{\mathrm{a}}$.). Quando indagadas sobre desrespeito por parte de algum profissional que prestou assistência, a negativa teve destaque nas três RSs (91,4\% na $9^{\mathrm{a}}$., $94,8 \%$ na $10^{\mathrm{a}}$. e $92,1 \%$ na $17^{\mathrm{a}}$.). 
Tabela 4. Intercorrências no período gestacional nos serviços públicos de Saúde do Estado do Paraná, PR, 2020.

\begin{tabular}{|c|c|c|c|c|c|c|}
\hline \multirow[t]{3}{*}{ Variáveis } & \multicolumn{6}{|c|}{ Regionais de Saúde } \\
\hline & \multicolumn{2}{|c|}{$9^{a}$} & \multicolumn{2}{|c|}{$10^{\mathrm{a}}$} & \multicolumn{2}{|c|}{$17^{\mathrm{a}}$} \\
\hline & $\mathbf{n}$ & $\%$ & $\mathbf{n}$ & $\%$ & $\mathbf{n}$ & $\%$ \\
\hline \multicolumn{7}{|l|}{ Intercorrência gestacional } \\
\hline Sim & 172 & 43,3 & 133 & 34,5 & 136 & 31,7 \\
\hline Não & 225 & 56,7 & 252 & 65,5 & 293 & 68,3 \\
\hline \multicolumn{7}{|l|}{ Doença Hipertensiva } \\
\hline Sim & 20 & 5,0 & 27 & 7,0 & 35 & 8,2 \\
\hline Não & 377 & 95 & 358 & 93 & 394 & 91,8 \\
\hline \multicolumn{7}{|l|}{$\begin{array}{l}\text { Doença Clínica (cardiopatias, } \\
\text { endocrinopatias, ITU) }\end{array}$} \\
\hline $\operatorname{Sim}$ & 10 & 2,5 & 12 & 3,1 & 9 & 2,1 \\
\hline Não & 387 & 97,5 & 373 & 96,9 & 420 & 97,9 \\
\hline \multicolumn{7}{|l|}{ Trabalho parto prematuro } \\
\hline Sim & 8 & 2,0 & 15 & 3,9 & 14 & 3,3 \\
\hline Não & 389 & 98 & 370 & 96,1 & 415 & 96,7 \\
\hline \multicolumn{7}{|l|}{ Sangramento } \\
\hline $\operatorname{Sim}$ & 39 & 9,8 & 19 & 4,9 & 36 & 8,4 \\
\hline Não & 358 & 90,2 & 366 & 95,1 & 393 & 91,6 \\
\hline \multicolumn{7}{|l|}{ Placenta Prévia } \\
\hline Sim & 2 & 0,5 & 12 & 3,1 & 9 & 2,1 \\
\hline Não & 395 & 99,5 & 373 & 96,9 & 420 & 97,9 \\
\hline \multicolumn{7}{|l|}{$\begin{array}{l}\text { Informada Hospital referência } \\
\text { intercorrência }\end{array}$} \\
\hline Sim & 375 & 94,5 & 360 & 93,5 & 371 & 86,5 \\
\hline Não & 22 & 5,5 & 25 & 6,5 & 58 & 13,5 \\
\hline \multicolumn{7}{|l|}{ Serviço na intercorrência } \\
\hline UBS / UPA* & 40 & 33,9 & 25 & 21,2 & 47 & 34,3 \\
\hline Hospital & 75 & 63,6 & 88 & 74,6 & 81 & 59,1 \\
\hline Outros (ambulatório/maternidade) & 3 & 2,5 & 5 & 4,2 & 9 & 6,6 \\
\hline \multicolumn{7}{|l|}{$\begin{array}{l}\text { Avaliação atendimento } \\
\text { intercorrência }\end{array}$} \\
\hline Rápido/Resolutivo & 109 & 92,4 & 106 & 89,8 & 88 & 64,2 \\
\hline Demorado & 9 & 7,6 & 12 & 10,2 & 49 & 35,8 \\
\hline Total & 397 & 100,0 & 385 & 100,0 & 429 & 100,0 \\
\hline
\end{tabular}

\section{Discussão}

O MS, por meio da Rede Cegonha (Portaria $n^{\circ} 1.459,2011$ ), estipula ações voltadas para a atenção no período gravídico-puerperal, determinando o início precoce do pré-natal, ideal antes de 14 semanas de gestação, e o total de pelo menos 6 consultas, além de outras etapas envolvidas para atingir a qualidade de atendimento. Este estudo nos mostra que nas três RSs esses quesitos ocorreram conforme o esperado. É importante compararmos a adesão ao pré-natal, em que os números 
são quase a totalidade, com o início precoce do atendimento. Esse último apresentou queda no percentual nas três RSs, principalmente na $10^{\text {a. }}$ RS. As mulheres que tiveram intercorrências nesta regional tiveram o início após 14 semanas de gestação, fazendo-nos identificar a necessidade de melhorias nas ações de captação precoce desta região.

A não participação no pré-natal foi considerada pequena mas precisa ser tratada como agravante à saúde maternoinfantil. Para Cardoso et al. (2017), muitas vezes ocorre devido à descoberta tardia ou à própria negação da gravidez, à não valorização da assistência ofertada e até mesmo à dificuldade de acesso ao serviço de saúde. Este dado nos faz avaliar como estão sendo repassadas as informações e as orientações à população.

O local escolhido para a realização do pré-natal foi com maior frequência a rede pública, principalmente na $9^{\text {a. }}$ RS. Esta informação reforça a importância da estruturação da Atenção Primária à Saúde (APS) em executar assistência adequada, com profissionais interligados e aptos a realizar adequadamente a estratificação de risco dessas mulheres, em habitual, intermediário e alto risco. Essa assistência deve ser de maneira contínua, direcionando-a para o local mais apropriado de atendimento e mantendo o acompanhamento em paralelo, favorecendo um prognostico positivo para o binômio mãe/filho (Sociedade Beneficente Israelita Brasileira Albert Einstein [SBIBAE], 2019; Teles et al., 2019).

Nas três RSs analisadas, tivemos mais destaque em gestações de risco habitual, porém um número considerável de alto risco, principalmente na $10^{\mathrm{a}}$. RS, sendo essas mães em sua maioria com ocupação remunerada, demonstrando que isso não impede o acesso ao pré-natal, iniciado antes de 14 semanas de gestação. A informação quanto ao risco foi transmitida às gestantes em apenas metade da amostra, em todas as RSs, sendo efetuados pelo profissional de enfermagem ( $9^{\mathrm{a}}$. RS) ou médico $\left(10^{\mathrm{a} .}\right.$ e $\left.17^{\mathrm{a} .} \mathrm{RSs}\right)$, evidenciando uma lacuna relevante na valorização dos riscos por parte dos profissionais de saúde, uma vez que deve ser transmitido imediatamente à gestante, dando orientações pertinentes em cada situação.

O MS determina que gestantes de risco intermediário e alto risco devem ser assistidas preferencialmente em serviços especializados que possuam equipamentos e profissionais preparados, mantendo apenas um acompanhamento em paralelo na atenção primária (SBIBAE, 2019). A porcentagem de encaminhamentos a serviços especializados foi pequena em todas as RSs, principalmente na $9^{\text {a. }}$ RS porém a maioria deles eram gestações de risco habitual. Nesse sentido, o baixo índice de encaminhamentos não deve ser entendido como negligência por parte do serviço e sim, resolutividade do cuidado no nível primário conforme determina o protocolo vigente.

Vale ressaltar ainda a importância da descoberta precoce da gravidez concomitantemente ao início do pré-natal para que os encaminhamentos a serviços especializados possam ocorrer em tempo oportuno, favorecendo o período (Santos \& Soares, 2018). Neste Estudo, detectou-se que na $9^{\text {a. }}$ RS, na qual o número de encaminhamento foi menor, grande parte teve início da assistência antes de 14 semanas de gestação e realizaram 6 ou mais consultas, favorecendo o tempo para verificação de possíveis agravos. Entre as mulheres que não passaram por esse processo, a maioria não apresentou intercorrências durante a gestação, e poucas das que tiveram, eram de alto risco, fato que serve de alerta aos profissionais de saúde para aperfeiçoamento desses encaminhamentos uma vez que gestantes de risco devem ser assistidas em local apropriado.

O tempo de espera por serviço especializado foi menor na $9^{\mathrm{a}}$. RS, sendo rápido ou até mesmo imediato; já na $17^{\mathrm{a}}$. RS a espera ultrapassou 29 dias em quase metade da amostra, sendo elas gestantes de alto risco e intermediário, contradizendo o que o Governo Federal, pelo Programa Rede Cegonha, preconiza em relação ao acesso às consultas especializadas em tempo oportuno (Portaria ${ }^{\circ} 1.459,2011$ ).

$\mathrm{Na}$ tentativa de avaliar os motivos que dificultaram o acesso a serviços mais complexos de atenção, um estudo realizado em 4 grandes cidades brasileiras identificou que direcionamentos desnecessários geram impactos no atendimento das gestantes e levam à retenção incorreta na atenção especializada e/ou descontinuidade na APS, interrompendo o acompanhamento compartilhado entre UBS e especialista, que seria o desejado (Fernandes et al., 2020). Cabe assim a cada gestor conhecer o perfil de sua população, reconhecendo as necessidades principais dos ambulatórios de especialidades bem 
como elaborar estratégias que driblem as filas de espera, além do mais, outra vez, vê-se a importância de intensificar a capacitação das equipes envolvidas no processo.

Ainda em relação ao preconizado, as gestantes devem ser assistidas de maneira mais intensificada nas últimas semanas de gestação devido à maior probabilidade de intercorrências neste estágio, não sendo prudente que elas tenham um intervalo entre sua última consulta pré-natal e o parto, superior a uma semana (Portaria $\left.\mathrm{n}^{\circ} 1.459,2011\right)$. Na $9^{\text {a. }}$ RS, houve número considerável de gestantes que aguardaram mais que 15 dias até o momento do parto, sinalizando uma lacuna importante da assistência a essas mulheres, deixando-as vulneráveis às complicações inerentes ao final da gestação.

Esse quadro pode muitas vezes sobrecarregar as instituições de atendimento de urgências, favorecendo também a perda de vínculo da atenção primária com essas gestantes, haja vista que não existe alta de pré-natal, esse atendimento deve-se manter até a finalização do puerpério (Gonçalves et al., 2017).

No que tange à realização de colpocitologia oncótica, apenas na $17^{\text {a. }}$ RS, destacou a realização durante o pré-natal ou com menos de 1 ano de intervalo, o que aponta mais uma lacuna na assistência pré-natal.

Lembrando que muitas vezes essas mulheres terão apenas essa oportunidade para avaliação da saúde reprodutiva, durante toda sua vida e elas deixam então de receber atenção integral e qualificada como deveriam. Esse achado evidencia a focalização dos profissionais prenatalistas no cuidado, o que acaba por limitar a assistência holística à mulher. Vale lembrar que, na gravidez, devido ao desequilíbrio da flora vaginal aliado às mudanças hormonais, o câncer de colo de útero torna-se o mais comum entre os cânceres associados à gravidez, tudo isso agrupado ao desconhecimento e ao medo da mulher quanto ao exame, e o despreparo por parte dos profissionais colocam essas mulheres em situações iminentes de risco à saúde (Lima, Steger \& Pontes, 2019; Texeira, Santos, Sanches, Silva \& Cavalcante, 2019).

Um número significativo de gestantes apresentou algum tipo de intercorrência, com maior frequência na $9^{\mathrm{a}}$. RS. Esses agravos foram semelhantes aos apresentados na literatura, como sangramento, doença hipertensiva e acometimentos clínicos (cardio e endocrinopatias e, até mesmo, ITU) (Varela et al., 2017), sinalizando mais uma vez a necessidade de detecção precoce de injúrias que possam gerar internações hospitalares, favorecendo complicações maternas e fetais, além de aumentar custos na oferta de cuidados.

Em um estudo realizado em um município do Paraná, foi identificado que as patologias mencionadas estão entre as principais causas de internações no período gestacional, com a Taxa de Intercorrência Obstétrica (TIO) de 37,2\%, tendo maior ocorrência em atendimentos públicos, adolescentes ou gestantes com idade mais avançada e nível socioeconômico mais baixo (Falavina et al., 2017).

Grande parte das mulheres que necessitaram de atendimento em casos de intercorrências procurou o serviço hospitalar e o avaliou como rápido e resolutivo. Entretanto, uma proporção importante considerou demorado, em maior número na $17^{\mathrm{a}}$. RS, levantando mais uma fragilidade da assistência a esse grupo vulnerável, cabendo aos gestores replanejar o fluxograma de atendimentos bem como capacitar sua equipe de atendimento.

Diante da preocupação com a saúde materno-infantil, o calendário vacinal das gestantes passa por constantes atualizações, no momento são necessárias três vacinas: contra difteria e tétano, contra hepatite $\mathrm{B}$, e, sazonalmente, contra influenza A. O cumprimento dessa etapa está constantemente correlacionado com as desigualdades socioeconômicas, demonstrando que os menos favorecidos enfrentam barreiras relacionadas à aceitação das vacinas por falta de acesso às informações, seja por nível cultural ou assistência pré-natal iniciada tardiamente de acordo com Oliveira et al. (2020).

O Ministério da Saúde determina o ingresso ao acompanhamento odontológico das gestantes assim que elas sejam inseridas no programa de pré-natal (SBIBAE, 2019). No entanto, tivemos frequência baixa neste quesito, na $9^{\text {a. }}$ e na $10^{\text {a }}$ RS, contrariando essa determinação.

Tal recomendação é justificada pela susceptibilidade a acometimentos periodontais decorrentes das ações hormonais, 
e a não adesão a essa conduta pode estar relacionada em alguns momentos à limitação dos profissionais envolvidos, principalmente por haver estigmas de possíveis riscos que possam afetar os bebês por meio dos procedimentos odontológicos mas, por outro lado, a transmissão da informação por parte do serviço quanto ao direito de acesso para as gestantes ainda é falha (Silva et al., 2020).

O seguimento pré-natal é um espaço importantíssimo para a implementação da educação em saúde, já que nesse momento a mulher está mais aberta a orientações e até mesmo mudanças de hábitos (Gonçalves et al., 2017). Nesse contexto, a estruturação de grupos de gestantes é fundamental e pode auxiliar a equipe multiprofissional a direcionar temas particulares de cada área que certamente enriquecerão a assistência.

Porém, na presente Pesquisa, a participação nos grupos foi extremamente baixa em todas as RSs, mostrando grave lacuna na assistência. Além de perder oportunidade para compartilhar informações sobre diversos agravos que possam ocorrer e talvez ser minimizados simplesmente por meio do acesso às orientações, perde-se o momento oportuno de reforçar a referência para o parto, local de atendimento de urgências e, mais além, orientar quanto aos cuidados puerperais que são de extrema importância para finalizar positivamente o ciclo da gestação. Cabe os gestores analisar se os grupos de gestantes estão deixando de ser ofertados devido às limitações relacionadas ao quadro de funcionários e funções desempenhadas pelas equipes da APS.

O perfil das mulheres atendidas nos serviços analisados foi de adultas jovens (19 e 34 anos) porém algumas ainda se encontravam no período de adolescência, predispondo-as a riscos decorrentes da vulnerabilidade do momento (Farias et al., 2020; Santos et al., 2018).

Não menos importante é a visão das gestantes em relação à satisfação do atendimento prestado. Pesquisas mostram que a opinião do paciente é importante para a efetividade da qualidade da assistência, sendo possível o estabelecimento de vínculo e a facilidade de acesso (Prudêncio \& Mamede, 2018). A assistência pré-natal prestada nas três RSs teve opiniões favoráveis de grande parte das participantes quanto à satisfação com o atendimento.

Visualizamos que muitas mulheres negaram desrespeito por parte dos profissionais que as assistiram - ponto positivo a ser mantido e estimulado quanto a seus benefícios, principalmente no que envolve a conquista e o estabelecimento de vínculo e, até mesmo, os fatores facilmente evitáveis, relacionados à morbimortalidade materna e infantil, vindo a relevante abordagem sobre violência obstétrica a ser reconhecida pela OMS (2014 apud Lansky et al., 2019) como questão de saúde pública, que se define como qualquer ato de violência praticado por um profissional que envolve a mulher grávida, a parturiente ou a puérpera ou até mesmo seu bebê, sendo considerados diversas atitudes que vão desde maus tratos físicos, verbais ou psicológicos até procedimentos não consentidos pela mulher (Lansky et al., 2019).

Como limitação deste Estudo, por ser transversal, embora forneça uma visão mais aprofundada de uma determinada população, os dados encontrados podem ser semelhantes a outras regiões.

\section{Conclusão}

De modo geral, a assistência pré-natal tem sido executada dentro do contexto preconizado pelo MS por meio da Rede Cegonha, nas três RSs analisadas. Foi perceptível que a procura e a captação ocorreram em tempo oportuno, antes de 14 semanas de gestação.

O seguimento do pré-natal foi satisfatório, sendo reforçado nos achados de vacinação, na resolutividade da APS verificado por meio do baixo número de encaminhamentos e pela satisfação da população atendida, favorecendo certamente o desfecho positivo.

No entanto, algumas lacunas com impacto negativo no cuidado foram identificadas, como a ocorrência de gravidez na adolescência e ainda a baixa participação das mulheres nos grupos de gestantes. Esses achados nos mostram riscos em nível 
social que interferem diretamente no momento gravídico delas, sinalizando dessa forma a importância do olhar dos gestores para a atuação multiprofissional da assistência, envolvendo nesse caso serviço social e psicologia. Além do mais, deve-se investigar o verdadeiro motivo que impediu a frequência aos grupos de gestantes, haja vista que, com essa prática, é possível realizar promoção e prevenção à saúde do binômio mãe/filho, favorecendo certamente o êxito da assistência.

Outro achado relevante que influencia diretamente no cuidado foi a não participação e, até mesmo, o início tardio do pré-natal, que mesmo sendo identificado com frequência baixa no estudo mostrou que ainda existe necessidade de ações voltadas à captação precoce desse público-alvo pois esta influencia diretamente no tempo adequado dos encaminhamentos necessários, minimizando a ocorrência de agravos que favoreçam os riscos.

Foram identificadas fragilidades relacionadas ao seguimento do protocolo implantado pelo MS no que diz respeito ao esclarecimento sobre o risco às gestantes, à realização de citologia oncótica, à consulta odontológica, ao tempo de espera em serviços especializados e ao tempo de espera entre a última consulta pré-natal e o parto.

Dessa forma, este estudo oferece subsídios aos gestores para a readequação de protocolos, de fluxos de atendimento e, ainda, de capacitações direcionadas às equipes de atuação na saúde materna, despertando assim visão mais ampla, escuta ativa e direcionada à realidade individual de cada mulher, oferecendo dessa forma assistência pré-natal de qualidade que garanta o desfecho favorável à mãe bem como seu bebê.

É válido ressaltar que cabem novos estudos que busquem entender os determinantes das fragilidades e lacunas evidenciadas pelo diagnóstico realizado nesta pesquisa.

\section{Agradecimentos}

À $9^{\mathrm{a}}, 10^{\mathrm{a}} \mathrm{e} 17^{\mathrm{a}}$ Regionais de Saúde pela colaboração na realização desta pesquisa.

À Fundação Araucária pela bolsa de estudo durante a realização da pós-graduação.

\section{Referências}

Cardoso, M. R. de S., Júnior, J. H. de O., Santos, M. A. dos, Santos, N. F., \& Cariri, L. S. (2017). A atuação do enfermeiro na assistência pré-natal no âmbito do Sistema Único de Saúde (SUS). Anais do International Nursing Congress, Aracaju, SE, Brasil. http://portal.saude.pe.gov.br/sites/portal.saude.pe.gov.br/fil

Falavina, L. P., Oliveira, R. R. de, Melo, E. C., Varela, P. L. R., \& Mathias, T. A. de F. (2017). Hospitalização durante a gravidez segundo financiamento do parto: um estudo de base populacional. Revista da Escola de Enfermagem da USP, 52, 1-8. https://doi.org/10.1590/S1980-220X2017032403317

Farias, R. V., Soares, C. F. S. e, Araújo, R. da S., Almeida, V. R. S. de, Leitão, D. de S., Santos, J. S. dos, Santos, L. S., Nogueira, S. D. de A., Morais, A. C., \& Oliveira, C. B. F. (2020). Gravidez na adolescência e o desfecho da prematuridade: uma revisão integrativa de literatura. Revista Eletrônica Acervo Saúde, 1-10. https://doi.org/10.25248/reas.e3977.2020

Fernandes, J. A., Venâncio, S. I., Pasche, D. F., Da Silva, F. L. G., Aratani, N., Tanaka, O. Y., Sanine, P. R., \& De Sousa Campos, G. W. (2020). Avaliação da atenção à gestação de alto risco em quatro metrópoles brasileiras. Cadernos de Saude Publica, 36(5), 1-14. https://doi.org/10.1590/0102-311X00120519

Gonçalves, M. F., Teixeira, É. M. B., Silva, M. A. D. S., Corsi, N. M., Ferrari, R. A. P., Pelloso, S. M., \& Cardelli, A. A. M. (2017). Pré-natal: preparo para o parto na atenção primária à saúde no sul do Brasil. Revista Gaucha de Enfermagem, 38(3), 1-8. https://doi.org/10.1590/1983-1447.2017.03.2016-0063

Guimarães, W. S. G., Parente, R. C. P., Guimarães, T. L. F., \& Garnelo, L. (2018). Acesso e qualidade da atenção pré-natal na estratégia Saúde da Família: infraestrutura, cuidado e gestão. Cadernos de Saude Publica, 34(5), 1-13. https://doi.org/10.1590/0102-311x00110417

Lansky, S., Souza, K. V. de, Peixoto, E. R. de M., Oliveira, B. J., Diniz, C. S. G., Vieira, N. F., Cunha, R. de O., \& Friche, A. A. de L. (2019). Obstetric violence: influences of the senses of birth exhibition in pregnant women childbirth experience. Ciencia $e$ Saude Coletiva, 24(8), 2811-2824. https://doi.org/10.1590/1413-81232018248.30102017

Lima, V. C. A. de, Steger, J., \& Pontes, S. R. L. (2019). Enfrentamento da mulher com diagnóstico de câncer no período gestacional. Revista Vista et Sanita da Faculdade União Goyazes, 12(2), 128-133.

Oliveira, S. de C., Silva, T. P. R. da, Velásquez-Melendez, G., Mendes, L. L., Martins, E. F., \& Rezende, E. M. (2020). Desigualdades sociais e obstétricas e vacinação em gestantes. Revista Brasileira de Enfermagem, 73(Suppl 4), 1-7.

Pereira, A. S., Shitsuka, D. M., Parreira, F. J., \& Shitsuka, R. (2018). Metodologia da pesquisa científica UFSM. https://repositorio.ufsm.br/bitstream/handle/1/15824/Lic_Computacao_Metodologia-Pesquisa-Cientifica.pdf?sequence=1

Portaria $N^{o}$ 1.459, de 24 de junho de 2011. (2011). Institui, no âmbito do Sistema Único de Saúde - SUS - a Rede Cegonha. 
Research, Society and Development, v. 10, n. 2, e37010212543, 2021

(CC BY 4.0) | ISSN 2525-3409 | DOI: http://dx.doi.org/10.33448/rsd-v10i2.12543

https://bvsms.saude.gov.br/bvs/saudelegis/gm/2011/prt1459_24_06_2011.html

Prudêncio, P. S., \& Mamede, F. V. (2018). Avaliação do cuidado pré-natal na atenção primária a saúde na percepção da gestante. Revista Gaucha de Enfermagem, 39, e20180077. https://doi.org/10.1590/1983-1447.2018.20180077

Santos, A. K. S., \& Soares, M. Z. P. (2018, September 25). Perfil de encaminhamentos ao pré-natal de alto risco do município de Aracaju/SE. Scire Salutis, 8(1), 44-52. https://doi.org/10.6008/cbpc2236-9600.2018.001.0006

Santos, L. A. V., Lara, M. O., Lima, R. C. R., Rocha, A. F., Rocha, E. M., Glória, J. C. R., \& Ribeiro, G. de C. (2018). História gestacional e características da assistência pré-natal de puérperas adolescentes e adultas em uma maternidade do interior de Minas Gerais, Brasil. Ciencia e Saude Coletiva, 23(2), 617-625. https://doi.org/10.1590/1413-81232018232.10962016

Silva, C. C. da, Savian, C. M., Prevedello, B. P., Zamberlan, C., Dalpian, D. M., \& Santos, B. Z. dos. (2020). Acesso e utilização de serviços odontológicos por gestantes: revisão integrativa da literatura. Ciencia e Saude Coletiva, 25(3), 827-835. https://doi.org/10.1590/1413-81232020253.01192018

Sociedade Beneficente Israelita Brasileira Albert Einstein. (2019). Nota técnica para organização da rede de atenção à saúde com foco na atenção primária: saúde da mulher na gestação, parto e puerpério autores e colaboradores. Saúde da mulher na gestação, parto e puerpério. São Paulo: Hospital Israelita Albert Einstein: Ministério da Saúde. https://atencaobasica.saude.rs.gov.br/upload/arquivos/202001/03091259-nt-gestante-planificasus.pdf

Teles, P. A., Mello, E. C., Panobianco, M. S., Gozzo, T. D. O., Paterra, T. da S. V., \& Nunes, L. C. (2019). Diagnósticos de enfermagem mais prevalentes em gestantes de alto risco. Enfermagem Em Foco, 10(3), 119-125.

Texeira, L. D. M., Santos, A. A. P. dos, Sanches, M. E. T. de L., Silva, J. M. de O. e, \& Cavalcante, M. V. (2019). Exame preventivo para o câncer de colo durante a gravidez: experiências das gestantes. Revista Baiana de Enfermagem, 33, 1-8. https://doi.org/10.18471/rbe.v33.33698

Varela, P. L. R., Oliveira, R. R. de, Melo, E. C., \& Mathias, T. A. de F. (2017). Intercorrências na gravidez em puérperas brasileiras atendidas nos sistemas público e privado de saúde. Revista Latino-Americana de Enfermagem, 25, e2949. https://doi.org/10.1590/1518-8345.2156.2949 